\title{
The Orchid Flora of Gunung Ledang (Mount Ophir), Malaysia - 120 Years after Ridley
}

\author{
Farah Alia Nordin', Ahmad Sofiman Othman ${ }^{1 *}$, Nur Asyikin Zainudin ${ }^{1}$, Nur \\ 'Atiqah Khalil', Najidah Asi', Afifah Azmi', Khairul Nasirudin Abu Mangsor', \\ Mohd Sukor Harun' ${ }^{1}$ and Khairul Faizee Mohd Zin ${ }^{3}$ \\ ${ }^{1}$ School of Biological Sciences, Universiti Sains Malaysia, 11800 USM, Pulau Pinang, Malaysia \\ ${ }^{2}$ Medical Transfusion Unit, Hospital Universiti Sains Malaysia, 16150 Kubang Kerian, Kelantan, Malaysia \\ 3Johor National Park Corporation, Level 1, Bangunan Dato'Mohamed Salleh Perang, Kota Iskandar, 79100 \\ Johor Bahru, Johor, Malaysia
}

\begin{abstract}
A comprehensive assessment on the orchid flora of Gunung Ledang, Johor, Malaysia was carried out from 2012 to 2018 with the aim to re-evaluate the presence of orchid species listed by Ridley in his "Journal of the Straits Branch of the Royal Asiatic Society 35:1-28", published in 1901, after more than 100 years. The relevant account for comparison is also listed, noting that Ridley's historical collections were for the isolated group of hills commonly known as Gunung Ledang (Mount Ophir), while the collated item in Orchidaceae is part of catalogues for the whole of Peninsular Malaysia. After Ridley, no account on the orchid flora of Gunung Ledang has been properly given, particularly from the uppermost peak of the mountain, where many interesting plants and orchids are to be found there. This study identified 26 species or $67 \%$ were the same as those recorded by Ridley

ARTICLE INFO

Article history:

Received: 19 November 2020

Accepted: 15 February 2021

Published: 28 May 2021

DOI: https://doi.org/10.47836/pjtas.44.2.07

E-mail addresses:

farahalianordin@usm.my (Farah Alia Nordin)

sofiman@usm.my (Ahmad Sofiman Othman)

asyikinzainuddin@gmail.com (Nur Asyikin Zainudin)

nuratiqahkhalil@usm.my (Nur'Atiqah Khalil)

ida.asi52@yahoo.com (Najidah Asi)

afifahazmi93@gmail.com (Afifah Azmi)

khairul_nasirudin@usm.my (Khairul Nasirudin Abu Mangsor)

dvsukor@usm.my (Mohd Sukor Harun)

khairulfaizee82@gmail.com (Khairul Faizee Mohd Zin)

* Corresponding author (1901), and 65 species or $83 \%$ of Turner (1995) checklist of 270 species of orchids for the state of Malacca and Johor, including the common and widespread species to Peninsular Malaysia. By contribution, this paper provides an updated account on the diversity of orchids in Gunung Ledang, listing 122 species of orchids, of which eight are endemic to Peninsular Malaysia, two are hyper-endemic known only from Gunung Ledang, and 30 were recognised as new records. A comparison table of the
\end{abstract}


Farah Alia Nordin, Ahmad Sofiman Othman, Nur Asyikin Zainudin, Nur 'Atiqah Khalil, Najidah Asi, Afifah Azmi, Khairul Nasirudin Abu Mangsor, Mohd Sukor Harun and Khairul Faizee Mohd Zin

current findings against Ridley (1901) and Turner (1995) is provided which shows only 16 species were the same in all three studies.

Keywords: Diversity, Gunung Ledang, H. N. Ridley, I. M. Turner, Mount Ophir, Orchidaceae

\section{INTRODUCTION}

Gunung Ledang, or historically known as the fabled Mount Ophir is an isolated mountain range consisted of several main peaks, with the highest is Puncak Gunung Ledang that stands at $1267 \mathrm{~m}$ above sea level. The mountain range is accessible both from Asahan to the north and from Tangkak to the east, as Gunung Ledang is located within the border of Malacca and Johor.

Knowledge on the orchids of Gunung Ledang was first published by Ridley in 1901, and his records have served as a prominent reference on orchid species diversity of that high isolated mountain. Before Ridley, Gunung Ledang has been visited and explored by Cuming and Lobb (Ridley, 1901) who have made extensive orchid collections and brought down a number of dried specimens as well, yet no account of their collections nor indeed of their expeditions appear to be ever published.

Ridley identified 39 species mostly collected from the uppermost part of the range at the elevation of about 900-1200 m, and only referring to a few of rarer species at the lower levels. Most of Ridley's account consisted of species he collected from the northwest part of the mountain, ascending to the top of the highest peak which is Puncak
Gunung Ledang from Malacca via Bukit Besar and Lubuk Kedondong, where the mountain is accessible with safety in the early days.

Ridley explored and ascended to the main peak of Gunung Ledang from Malacca via two routes. The first route started from the densely wooded Bukit Besar where the ascent commences steeply towards Padang Batu at about 823 m elevation, a large sloping rock-face covered in part with thick grass and sedges, among which grow gnarled montane trees such as Baeckia, Leptospermum, and Podocarpus, with orchids such as Arundina graminifolia, Spathoglottis aurea, and Paphiopedilum barbatum heavily populated the damp spots. However, the population of $S$. aurea in Gunung Ledang seems to be rare nowadays. He then marched through Gunung Tunduk, a large bare rock area which a good view of Malacca is obtainable, before not far off descended into a damp valley, and continued by a stiff steep climb to reach the extreme peak of Gunung Ledang. The second route taken by Ridley was via a lower peak of the range known as Gunung Mering, where he ascended with Mr. Hervey in 1892 from Lubuk Kedondong. Ridley reported that Gunung Mering is a less visited peak which claimed by local people as inaccessible, even though the ascent proved to be just merely stiff climbs that in parts require one to cross some smooth steep rocks. About halfway up Gunung Mering, they arrived at a point across Padang Batu, a stone field with a cascading stream and were surrounded by cliffs which were covered with forest. At 
present day, both Ridley's historical routes he ascended from Malacca via Lubuk Kedondong are now known as the 'Asahan Trail', named after a small town in Jasin that is located within the border of three states. Thus, from now onwards throughout this manuscript, Ridley's historical routes will be consistently cited as Asahan Trail, referring to the same routes taken but with new designation.

During Ridley's day, large part of Gunung Ledang was left unvisited, particularly to the east and south parts of the mountain range that lies within the state of Johor, very likely due to inaccessibility and safety reasons. Nowadays, Gunung Ledang is accessible from Tangkak in the southeast and Jementah in the northwest. The route from Tangkak via Sagil is popular among avid hikers, as ascending the Lagenda Trail to the top of Gunung Ledang is challenging but less arduous in comparison to Asahan. At the beginning of the trail, the route is quite treacherous with protruding tree roots, huge rocky boulders, and dense forest canopy. The ascensions commence starting from Batu Orkid towards Bukit Botak, where at some points, requiring one to scale up using ladders and ropes. From Bukit Botak towards Anjung Mahligai (1061 $\mathrm{m}$ above sea level), there is a clearing where intermittently covered in mist during the day, the surrounding is beautiful and the summit of Gunung Ledang is within sight. In contrast, the route from Jementah over Ulu Jementah Trail is seldom visited due to the long-winded mountain ridge and extremely steep ascent along the way through several lower peaks such as from Puncak Jementah (945 m above sea level) to Gunung Mahligai (1236 m above sea level) before summiting Gunung Ledang. Water supply is also scarce; a stream with small running water is only accessible at the first $2 \mathrm{~km}$ from the trail entrance, and next possible water sources will come from small creeks running through rocks. Most part of the trail is covered in dense lowland and hill forest canopy, untouched, and a best place for the botanical study of many plant groups. No account on the orchid flora from this part of Gunung Ledang has ever been published, thus the accomplishment in preparing the updates will augment the fundamental knowledge that has been firmly set up by Ridley.

The relevant account for comparison by Turner (1995) is also listed, noting that Ridley's historical collections were for the isolated group of hills commonly known as Gunung Ledang, while Turner's item in Orchidaceae is part of his catalogues for the whole of Peninsular Malaysia. In 1995, Turner published his checklist "A Catalogue of the Vascular Plants of Malaya" (in The Gardens' Bulletin Singapore 47:2), an encyclopaedic compilation from his assiduous examination on the herbarium specimens holding of the Singapore Botanical Garden's Herbarium (SING), the Royal Botanic Gardens at Kew (K), and local herbaria in the Forest Research Institute of Malaysia (KEP), University of Malaya (KLU), Biology Department, Universiti Putra Malaysia (UPM), and Universiti Kebangsaan Malaysia (UKMB). 
In his checklist, Turner listed 129 species of orchids specifically known from Malacca and Johor, with an additional of 141 species recognized as common and widespread throughout Peninsular Malaysia. He mentioned two hyper-endemic species known only from Gunung Ledang, Hetaeria elegans Ridl., which Ridley discovered from Gunung Tunduk and described it in 1908; and Anoectochilus burmannicus Rolfe, which is only known from one locality in the Malay Peninsula.

\section{MATERIALS AND METHODS}

Ridley's historical routes in Asahan were revisited, with two new routes accessible from Tangkak and Jementah were visited in this study (Figure 1). Seven forest trails were explored and assessed which are, (i) Asahan Trail via Lubuk Kedondong, (ii) Asahan
Trail via Dataran Damai Waterfall (Gunung Mering), (iii) Lagenda Trail via Batu Orkid, (iv) Ayer Panas Trail via Kolam Gajah, (v) Ulu Jementah Trail via Jeram Tinggi, (vi) Gunung Mahligai, and (vii) Gunung Ledang. The collections were made based on convenient sampling method along the seven forest trails from base of the foothill ascent to the peak of Gunung Ledang and vice versa. Observations were also made along the tarred road starting from the Taman Hutan Lagenda Park Office towards the Telekom Tower, which is located 500 $\mathrm{m}$ from the main peak of Gunung Ledang.

The specimens were identified using the morphological characters described, and the identification keys prepared by Comber (2001), Go et al. (2015), Holttum (1964), Ridley (1907, 1924), Seidenfaden and Smitinand (1959) as well as Seidenfaden and

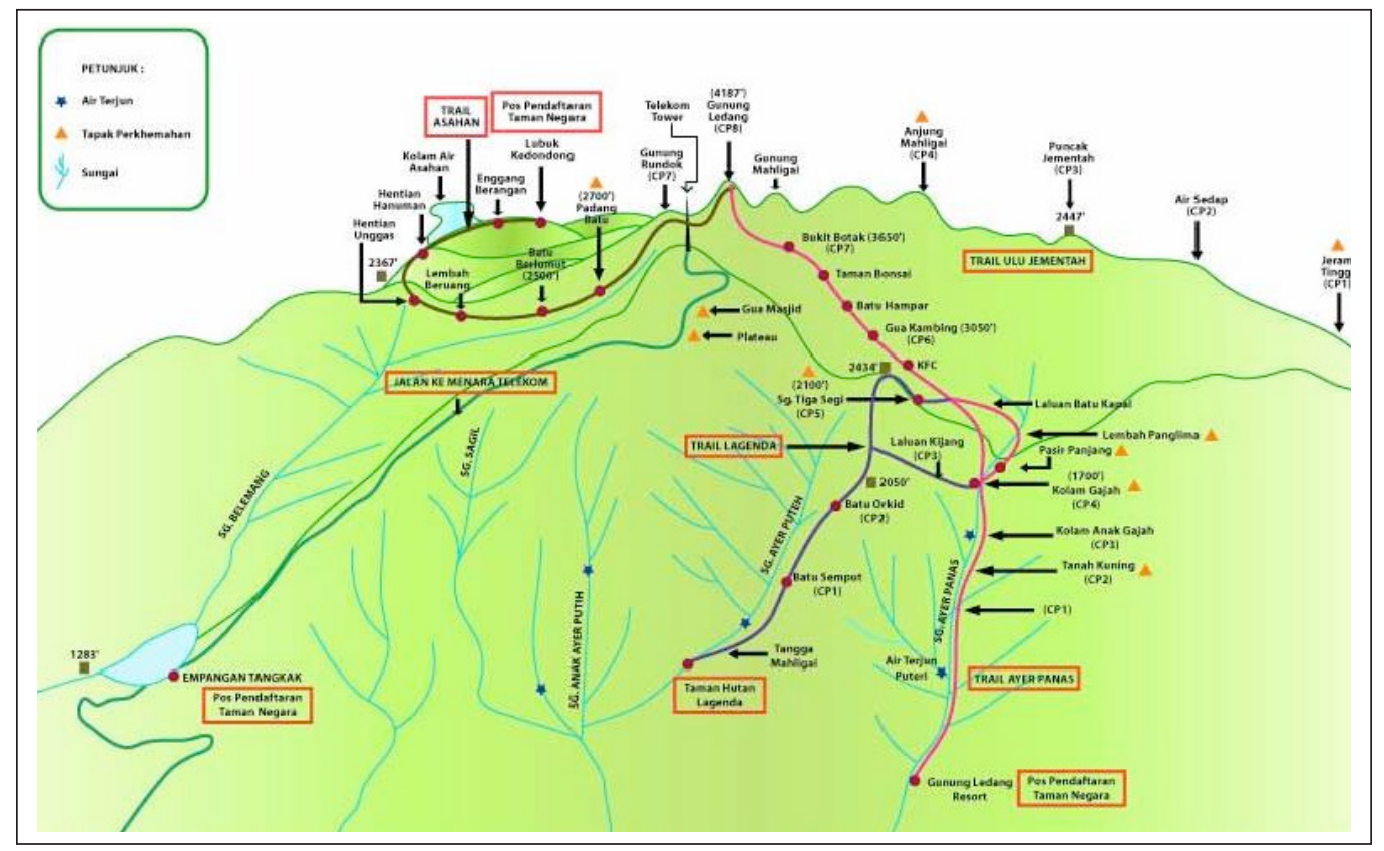

Figure 1. Trails in Gunung Ledang. Map courtesy of Johor National Park Corporation 
Wood (1992). The current accepted species name will be validated through the updated online database - Kew World Checklist of Selected Plant Families (WCSP) (2020), and the checklist made available by Ong et al. (2017).

Observations on the vegetative and reproductive macromorphological characteristics of species under investigation would be conducted in the fields. The geographical, ecological, and geological attributes would also be recorded. For detail micromorphological characteristics of selected taxa, specimens would be examined under field and stereo microscopes. Most of the plant specimens would be documented in the form of photographs, and collection of living specimens was to be circumvented in any way possible, as an approach for species as well as habitat preservation and conservation. Preserved herbarium specimens and spirit materials of the taxa collected would be deposited in Herbarium of School of Biological Sciences, Universiti Sains Malaysia (USM). Living collections for germplasm studies and exsitu conservation purposes would be cultivated in Taman Flora, School of Biological Sciences, USM.

\section{RESULTS}

From this study, a total of 104 orchid species from 62 genera have been identified from Gunung Ledang, of which five are endemic to Peninsular Malaysia, 30 were recognised as new records to the locality, and one undetermined species. The list also serves for comparison with the works by Ridley and by Turner in Malacca and Johor (Table $1)$.

Table 1

Species list: Comparison of orchid species found in Gunung Ledang since Ridley (1901)

\begin{tabular}{|c|c|c|c|c|}
\hline No. & Species & Ridley (1901) & Turner (1995) & Current study \\
\hline 1 & Acriopsis liliifolia (J.Köenig) Ormerod var. liliifolia & & $\sqrt{1}$ & $\sqrt{ }$ \\
\hline 2 & Aerides odorata Lour. & & $\sqrt{ }$ & $\sqrt{ }$ \\
\hline 3 & Agrostophyllum stipulatum (Griff.) Schltr. subsp. stipulatum & $\sqrt{ }$ & & $\sqrt{ }$ \\
\hline 4 & Anoectochilus albolineatus C.S.P.Parish \& Rchb.f. & & $\sqrt{ }$ & $\sqrt{ }$ \\
\hline 5 & Anoectochilus geniculatus Ridl. & $\sqrt{ }$ & & \\
\hline 6 & **Anoectochilus burmannicus Rolfe & & $\sqrt{ }$ & \\
\hline 7 & Anoectochilus sp. & & & $\sqrt{ }$ \\
\hline 8 & Apostasia latifolia Rolfe & $\sqrt{ }$ & $\sqrt{ }$ & $\sqrt{ }$ \\
\hline 9 & Apostasia nuda $\mathrm{R} . \mathrm{Br}$. in N.Wallich & $\sqrt{ }$ & $\sqrt{ }$ & $\sqrt{ }$ \\
\hline 10 & Apostasia wallichii R.Br. in N.Wallich & & & $\sqrt{ }$ \\
\hline 11 & Appendicula anceps Blume & & $\sqrt{ }$ & $\sqrt{ }$ \\
\hline 12 & Appendicula cornuta Blume & & $\sqrt{ }$ & $\sqrt{ }$ \\
\hline 13 & Appendicula reflexa Blume & & $\sqrt{ }$ & $\sqrt{ }$ \\
\hline 14 & Arundina graminifolia (D.Don) Hochr. & $\sqrt{ }$ & $\sqrt{ }$ & $\sqrt{ }$ \\
\hline 15 & Bromheadia aporoides Rchb.f. & $\sqrt{ }$ & & \\
\hline 16 & Bromheadia alticola Ridl. & $\sqrt{ }$ & $\sqrt{ }$ & \\
\hline 17 & Bromheadia brevifolia Ridl. & & $\sqrt{ }$ & \\
\hline 18 & Bromheadia finlaysoniana (Lindl.) Miq. & & $\sqrt{ }$ & $\sqrt{ }$ \\
\hline 19 & *Bromheadia pungens Ridl. & $\sqrt{ }$ & $\sqrt{ }$ & $\sqrt{ }$ \\
\hline 20 & *Bromheadia rupestris Ridl. & $\sqrt{ }$ & $\sqrt{ }$ & $\sqrt{ }$ \\
\hline 21 & Bromheadia truncata Seidenf. & & $\sqrt{ }$ & $\sqrt{ }$ \\
\hline 22 & Bulbophyllum clandestinum Lindl. & & $\sqrt{ }$ & $\sqrt{ }$ \\
\hline
\end{tabular}


Farah Alia Nordin, Ahmad Sofiman Othman, Nur Asyikin Zainudin, Nur 'Atiqah Khalil, Najidah Asi, Afifah Azmi, Khairul Nasirudin Abu Mangsor, Mohd Sukor Harun and Khairul Faizee Mohd Zin

Table 1 (continue)

\begin{tabular}{|c|c|c|c|c|}
\hline No. & Species & Ridley (1901) & Turner (1995) & Current study \\
\hline 22 & Bulbophyllum clandestinum Lindl. & & $\sqrt{ }$ & $\sqrt{ }$ \\
\hline 23 & Bulbophyllum elevatopunctatum J.J.Sm. & & & $\sqrt{ }$ \\
\hline 24 & Bulbophyllum fenestratum J.J.Sm. & & & $\sqrt{ }$ \\
\hline 25 & Bulbophyllum gracillimum (Rolfe) Rolfe & & $\sqrt{ }$ & $\sqrt{ }$ \\
\hline 26 & Bulbophyllum purpurascens Teijism. \& Binn. & $\sqrt{ }$ & $\sqrt{ }$ & $\sqrt{ }$ \\
\hline 27 & Bulbophyllum pustulatum Ridl. & & $\sqrt{ }$ & \\
\hline 28 & Bulbophyllum striatellum Ridl. & & $\sqrt{ }$ & $\sqrt{ }$ \\
\hline 29 & Bulbophyllum uniflorum (Blume) Hassk. & & $\sqrt{ }$ & $\sqrt{ }$ \\
\hline 30 & Bulbophyllum vaginatum (Lindl.) Rchb.f. in W.G.Walpers & $\sqrt{ }$ & $\sqrt{ }$ & $\sqrt{ }$ \\
\hline 31 & $\begin{array}{l}\text { Campanulorchis pellipes (Rchb.f. ex Hook.f.) Y.P.Ng \& } \\
\text { P.J.Cribb }\end{array}$ & $\sqrt{ }$ & $\sqrt{ }$ & $\sqrt{ }$ \\
\hline 32 & Ceratostylis ampullacea Kraenzl. & & $\sqrt{ }$ & $\sqrt{ }$ \\
\hline 33 & Ceratostylis gracilis Blume & $\sqrt{ }$ & & \\
\hline 34 & Ceratostylis subulata Blume & & $\sqrt{ }$ & $\sqrt{ }$ \\
\hline 35 & Calanthe angustifolia (Blume) Lindl. & $\sqrt{ }$ & & $\sqrt{ }$ \\
\hline 36 & Claderia viridiflora Hook.f. & $\sqrt{ }$ & $\sqrt{ }$ & $\sqrt{ }$ \\
\hline 37 & Cleisostoma suffusum (Ridl.) Garay & & $\sqrt{ }$ & \\
\hline 38 & *Coelogyne anceps Hook.f. & & & $\sqrt{ }$ \\
\hline 39 & Coelogyne cumingii Lindl. & $\sqrt{ }$ & $\sqrt{ }$ & \\
\hline 40 & *Coelogyne kaliana P.J.Cribb & & & $\sqrt{ }$ \\
\hline 41 & Coelogyne testacea Lindl. & & $\sqrt{ }$ & $\sqrt{ }$ \\
\hline 42 & Coelogyne tomentosa Lindl. & $\sqrt{ }$ & & $\sqrt{ }$ \\
\hline 43 & Corybas carinatus (J.J.Sm.) Schltr. & & $\sqrt{ }$ & $\sqrt{ }$ \\
\hline 44 & Corymborkis veratrifolia (Reinw.) Blume & & $\sqrt{ }$ & $\sqrt{ }$ \\
\hline 45 & Crepidium calophyllum (Rchb.f.) Szlach. & & $\sqrt{ }$ & $\sqrt{ }$ \\
\hline 46 & Cryptostylis arachnites (Blume) Hassk. in C.L.Blume & & $\sqrt{ }$ & $\sqrt{ }$ \\
\hline 47 & Cylindrolobus nutans (Lindl.) J.J.Wood & $\sqrt{ }$ & $\sqrt{ }$ & $\sqrt{ }$ \\
\hline 48 & Cymbidium finlaysonianum Lindl. & & & $\sqrt{ }$ \\
\hline 49 & Dendrobium angustifolium (Blume) Lindl. & & $\sqrt{ }$ & $\sqrt{ }$ \\
\hline 50 & Dendrobium convexum (Blume) Lindl. & & $\sqrt{ }$ & $\sqrt{ }$ \\
\hline 51 & Dendrobium crumenatum Sw. & & $\sqrt{ }$ & $\sqrt{ }$ \\
\hline 52 & Dendrobium derryi Ridl. & & & $\sqrt{ }$ \\
\hline 53 & Dendrobium geminatum (Blume) Lindl. & & & $\sqrt{ }$ \\
\hline 54 & Dendrobium indivisum (Blume) Miq. & & $\sqrt{ }$ & $\sqrt{ }$ \\
\hline 55 & *Dendrobium kelsallii Ridl. & $\sqrt{ }$ & & \\
\hline 56 & Dendrobium lamellatum (Blume) Lindl. & & & $\sqrt{ }$ \\
\hline 57 & Dendrobium leonis (Lindl.) Rchb.f. in W.G.Walpers & & $\sqrt{ }$ & $\sqrt{ }$ \\
\hline 58 & Dendrobium longipes Hook.f. & & & $\sqrt{ }$ \\
\hline 59 & Dendrobium macropodum Hook.f. & & & $\sqrt{ }$ \\
\hline 60 & Dendrobium mannii Ridl. & & $\sqrt{ }$ & \\
\hline 61 & Dendrobium metachilinum Rchb.f. & & $\sqrt{ }$ & $\sqrt{ }$ \\
\hline 62 & Dendrobium pachyglossum E.C.Parish \& Rchb.f. & & & $\sqrt{ }$ \\
\hline 63 & Dendrobium uniflorum Griff. & $\sqrt{ }$ & $\sqrt{ }$ & $\sqrt{ }$ \\
\hline 64 & Dendrobium villosulum Wall. ex Lindl. & $\sqrt{ }$ & $\sqrt{ }$ & $\sqrt{ }$ \\
\hline 65 & Dendrochilun linearifolium Hook.f. & $\sqrt{ }$ & & $\sqrt{ }$ \\
\hline 66 & Dendrochilum longifolium Rchb.f. & & & $\sqrt{ }$ \\
\hline 67 & Dipodium conduplicatum J.J.Sm. & & & $\sqrt{ }$ \\
\hline 68 & Erythrodes latifolia Blume & & & $\sqrt{ }$ \\
\hline 69 & Erythrorchis altissima (Blume) Blume & & & $\sqrt{ }$ \\
\hline 70 & Galeola nudifolia Lour. & & $\sqrt{ }$ & $\sqrt{ }$ \\
\hline 71 & Gastrodia javanica (Blume) Lindl. & & & $\sqrt{ }$ \\
\hline 72 & Geodorum densiflorum (Lam.) Schltr. & & $\sqrt{ }$ & $\sqrt{ }$ \\
\hline 73 & Goodyera rubicunda (Blume) Lindl. & & $\sqrt{ }$ & $\sqrt{ }$ \\
\hline 74 & Goodyera viridiflora (Blume) Blume & & & $\sqrt{ }$ \\
\hline
\end{tabular}


Table 1 (continue)

\begin{tabular}{|c|c|c|c|c|}
\hline No. & Species & Ridley (1901) & Turner (1995) & Current study \\
\hline 75 & Grammatophyllum speciosum Blume & & $\sqrt{ }$ & $\sqrt{ }$ \\
\hline 76 & Habenaria rhodocheila Hance & & & $\sqrt{ }$ \\
\hline 77 & Hetaeria elata Hook.f. & $\sqrt{ }$ & & $\sqrt{ }$ \\
\hline 78 & **Hetaeria elegans Ridl. & $\sqrt{ }$ & $\sqrt{ }$ & \\
\hline 79 & Hylophila mollis Lindl. & & $\sqrt{ }$ & $\sqrt{ }$ \\
\hline 80 & Lecanorchis malaccensis Ridl. & & $\sqrt{ }$ & $\sqrt{ }$ \\
\hline 81 & Liparis barbata Lindl. & & $\sqrt{ }$ & $\sqrt{ }$ \\
\hline 82 & Liparis elegans Lindl. & $\sqrt{ }$ & $\sqrt{ }$ & $\sqrt{ }$ \\
\hline 83 & Liparis maingayi (Hook.f.) Ridl. & $\sqrt{ }$ & $\sqrt{ }$ & \\
\hline 84 & Liparis viridicallus Holttum & & & $\sqrt{ }$ \\
\hline 85 & Luisia sp. & & & $\sqrt{ }$ \\
\hline 86 & Macodes petola (Blume) Lindl. & $\sqrt{ }$ & & $\sqrt{ }$ \\
\hline 87 & Neuwiedia griffithii Rchb.f. & & $\sqrt{ }$ & $\sqrt{ }$ \\
\hline 88 & Neuwiedia veratrifolia Blume & & & $\sqrt{ }$ \\
\hline 89 & *Oberonia bertoldii King \& Pantl. & & $\sqrt{ }$ & $\sqrt{ }$ \\
\hline 90 & Paphiopedilum barbatum (Lindl.) Pfitzer & $\sqrt{ }$ & $\sqrt{ }$ & $\sqrt{ }$ \\
\hline 91 & Peristylus maingayi (King \& Pantl.) J.J.Wood \& Ormerod & & $\sqrt{ }$ & $\sqrt{ }$ \\
\hline 92 & Peristylus monticola (Ridl.) Seidenf. & $\sqrt{ }$ & $\sqrt{ }$ & $\sqrt{ }$ \\
\hline 93 & Phalaenopsis deliciosa Rchb.f. & & & $\sqrt{ }$ \\
\hline 94 & Phalaenopsis fuscata Rchb.f. & & & $\sqrt{ }$ \\
\hline 95 & Pholidota carnea (Blume) Lindl. var. carnea & & & $\sqrt{ }$ \\
\hline 96 & *Pinalia atrovinosa (Carr) Schuit. & & $\sqrt{ }$ & \\
\hline 97 & Pinalia bractescens (Lindl.) Kuntze & & $\sqrt{ }$ & $\sqrt{ }$ \\
\hline 98 & Platanthera angustata (Blume) Lindl. & $\sqrt{ }$ & & $\sqrt{ }$ \\
\hline 99 & Plocoglottis javanica Blume & & $\sqrt{ }$ & $\sqrt{ }$ \\
\hline 100 & Podochilus microphyllus Lindl. & $\sqrt{ }$ & $\sqrt{ }$ & $\sqrt{ }$ \\
\hline 101 & Pomatocalpa diffusum Breda & & $\sqrt{ }$ & $\sqrt{ }$ \\
\hline 102 & Renanthera histrionica Rchb.f. & $\sqrt{ }$ & & $\sqrt{ }$ \\
\hline 103 & Rhynchostylis sp. & & & $\sqrt{ }$ \\
\hline 104 & Spathoglottis aurea Lindl. & $\sqrt{ }$ & $\sqrt{ }$ & \\
\hline 105 & Spathoglottis plicata Blume & & $\sqrt{ }$ & $\sqrt{ }$ \\
\hline 106 & Stichorkis gibbosa (Finet) J.J.Wood & & $\sqrt{ }$ & $\sqrt{ }$ \\
\hline 107 & Strongyleria pannea (Lindl.) Schuit. & & $\sqrt{ }$ & $\sqrt{ }$ \\
\hline 108 & Tainia maingayi Hook.f. & $\sqrt{ }$ & & \\
\hline 109 & Thecopus maingayi (Hook.f.) Seidenf. & & $\sqrt{ }$ & $\sqrt{ }$ \\
\hline 110 & Tainia speciosa Blume & $\sqrt{ }$ & $\sqrt{ }$ & $\sqrt{ }$ \\
\hline 111 & Thrixspermum sp. & & & $\sqrt{ }$ \\
\hline 112 & Trichotosia ferox Blume & & $\sqrt{ }$ & $\sqrt{ }$ \\
\hline 113 & Trichotosia gracilis (Hook.f.) Kraenzl. & & $\sqrt{ }$ & $\sqrt{ }$ \\
\hline 114 & Trichotosia pauciflora Blume & $\sqrt{ }$ & $\sqrt{ }$ & \\
\hline 115 & Trichotosia poculata (Ridl.) Kraenzl. & & $\sqrt{ }$ & $\sqrt{ }$ \\
\hline 116 & Trichotosia velutina (Lodd. ex Lindl.) Kraenzl. & $\sqrt{ }$ & $\sqrt{ }$ & \\
\hline 117 & Trichotosia vestita (Wall. ex Lindl.) Kraenzl. & $\sqrt{ }$ & $\sqrt{ }$ & \\
\hline 118 & Tropidia angulosa (Lindl.) Blume & & & $\sqrt{ }$ \\
\hline 119 & Tropidia curculigoides Lindl. & $\sqrt{ }$ & $\sqrt{ }$ & $\sqrt{ }$ \\
\hline 120 & Vanilla griffithii Rchb.f. & & $\sqrt{ }$ & $\sqrt{ }$ \\
\hline 121 & Zeuxine affinis (Lindl.) Benth. ex Hook.f. & & & $\sqrt{ }$ \\
\hline \multirow[t]{2}{*}{122} & Zeuxine gracilis (Breda) Blume & & & $\sqrt{ }$ \\
\hline & Total & 39 & 78 & 104 \\
\hline
\end{tabular}

Note.

$\sqrt{ }$ Present

* Endemic to Peninsular Malaysia as reported by Turner (1995) and Ong et al. (2017)

** Hyper-endemic species to Gunung Ledang as reported by Turner (1995) 
Farah Alia Nordin, Ahmad Sofiman Othman, Nur Asyikin Zainudin, Nur 'Atiqah Khalil, Najidah Asi,

Afifah Azmi, Khairul Nasirudin Abu Mangsor, Mohd Sukor Harun and Khairul Faizee Mohd Zin

The finding has identified 26 species or $67 \%$ were the same as those recorded by Ridley (1901), and 65 species or $83 \%$ of Turner (1995) checklist of 270 species of orchids for Johor, Malacca, and the whole of Peninsular Malaysia. Only 16 species were the same in all three studies, and 18 species which were listed before by Ridley and Turner were not recollected in this study, including the two hyper-endemics to Gunung Ledang, Hetaeria elegans, and Anoectochilus burmannicus. Checklist on the orchid species found in Gunung Ledang is provided as in Table 2 .

\section{Table 2}

Checklist of Gunung Ledang orchids and the area of occurrences in Peninsular Malaysia: 122 species (from 1901-present)

\begin{tabular}{|c|c|}
\hline No. & Species \\
\hline 1 & $\begin{array}{l}\text { Acriopsis liliifolia (J.Köenig) Ormerod var. liliifolia } \\
\text { (Synonym: Acriopsis javanica Reinw. ex Blume) } \\
\text { General Distribution: Sikkim to North West Pacific } \\
\text { Distribution in Peninsular Malaysia: Common in lowlands throughout } \\
\text { Lifeform: Epiphytic }\end{array}$ \\
\hline 2 & $\begin{array}{l}\text { Aerides odorata Lour. } \\
\text { General Distribution: China (W. Yunnan, Guangdong) to Tropical Asia } \\
\text { Distribution in Peninsular Malaysia: Common in lowlands throughout } \\
\text { Lifeform: Epiphytic }\end{array}$ \\
\hline 3 & $\begin{array}{l}\text { Agrostophyllum stipulatum (Griff.) Schltr. subsp. stipulatum } \\
\text { General Distribution: Indo-China, Malesia to Solomon Island } \\
\text { Distribution in Peninsular Malaysia: Johor; lowland and montane forest } \\
\text { Lifeform: Epiphytic }\end{array}$ \\
\hline 4 & $\begin{array}{l}\text { Anoectochilus albolineatus C.S.P.Parish \& Rchb.f. } \\
\text { General Distribution: Indo-China } \\
\text { Distribution in Peninsular Malaysia: Widespread; montane forest at 1000-1300 m } \\
\text { Lifeform: Terrestrial }\end{array}$ \\
\hline 5 & $\begin{array}{l}\text { Anoectochilus geniculatus Ridl. } \\
\text { General Distribution: Myanmar to West Malesia } \\
\text { Distribution in Peninsular Malaysia: Widespread; hill and montane forest } \\
\text { Lifeform: Terrestrial }\end{array}$ \\
\hline 6 & $\begin{array}{l}\text { Anoectochilus burmannicus Rolfe } \\
\text { General Distribution: China (S. Yunnan) to Pen. Malaysia } \\
\text { Distribution in Peninsular Malaysia: Known only from Gunung Ledang, Johor; montane forest } \\
\text { Lifeform: Terrestrial }\end{array}$ \\
\hline 7 & $\begin{array}{l}\text { Anoectochilus sp. (NAJ 17) } \\
\text { Distribution in Peninsular Malaysia: Gunung Ledang, Johor; montane forest at } 1000 \mathrm{~m} \\
\text { Lifeform: Terrestrial }\end{array}$ \\
\hline 8 & $\begin{array}{l}\text { Apostasia latifolia Rolfe } \\
\text { General Distribution: West Malesia } \\
\text { Distribution in Peninsular Malaysia: Perak, Pahang, Melaka; hill and montane forest } \\
\text { Lifeform: Terrestrial }\end{array}$ \\
\hline 9 & $\begin{array}{l}\text { Apostasia nuda } \text { R.Br. in N.Wallich } \\
\text { General Distribution: Assam to West Malesia } \\
\text { Distribution in Peninsular Malaysia: Widespread; lowland and hill forest to } 900 \mathrm{~m} \\
\text { Lifeform: Terrestrial }\end{array}$ \\
\hline 10 & $\begin{array}{l}\text { Apostasia wallichii R.Br. in N.Wallich } \\
\text { General Distribution: Japan (Island of Kyushu), China (South West Yunnan) } \\
\text { to Tropical Asia and North Australia } \\
\text { Distribution in Peninsular Malaysia: Scattered localities; lowland forest to } 600 \mathrm{~m} \\
\text { Lifeform: Terrestrial }\end{array}$ \\
\hline
\end{tabular}


Table 2 (continue)




Farah Alia Nordin, Ahmad Sofiman Othman, Nur Asyikin Zainudin, Nur 'Atiqah Khalil, Najidah Asi, Afifah Azmi, Khairul Nasirudin Abu Mangsor, Mohd Sukor Harun and Khairul Faizee Mohd Zin

Table 2 (continue)

\begin{tabular}{|c|c|}
\hline No. & Species \\
\hline \multirow[t]{4}{*}{25} & Bulbophyllum gracillimum (Rolfe) Rolfe \\
\hline & General Distribution: Peninsula Thailand to Southwest Pacific \\
\hline & Distribution in Peninsular Malaysia: Common throughout; lowland forest \\
\hline & Lifeform: Epiphytic and lithophytic \\
\hline \multirow[t]{4}{*}{26} & Bulbophyllum purpurascens Teijism. \& Binn. \\
\hline & General Distribution: Peninsula Thailand to West Malesia \\
\hline & Distribution in Peninsular Malaysia: Common throughout; lowland forest \\
\hline & Lifeform: Epiphytic and lithophytic \\
\hline \multirow[t]{4}{*}{27} & Bulbophyllum pustulatum Ridl. \\
\hline & General Distribution: Peninsular Malaysia and Borneo \\
\hline & Distribution in Peninsular Malaysia: Johor; lowland forest \\
\hline & Lifeform: Epiphytic \\
\hline \multirow[t]{4}{*}{28} & Bulbophyllum striatellum Ridl. \\
\hline & General Distribution: Peninsular Malaysia and Borneo (Sabah, Sarawak) \\
\hline & Distribution in Peninsular Malaysia: Kelantan and Johor; lowland forest \\
\hline & Lifeform: Epiphytic \\
\hline \multirow[t]{4}{*}{29} & Bulbophyllum uniflorum (Blume) Hassk. \\
\hline & General Distribution: West and Central Malesia \\
\hline & Distribution in Peninsular Malaysia: Widespread; montane forest \\
\hline & Lifeform: Epiphytic \\
\hline \multirow[t]{4}{*}{30} & Bulbophyllum vaginatum (Lindl.) Rchb.f. in W.G.Walpers \\
\hline & General Distribution: Peninsula Thailand to West Malesia \\
\hline & Distribution in Peninsular Malaysia: Common and widespread; lowland forest \\
\hline & Lifeform: Epiphytic \\
\hline \multirow[t]{4}{*}{31} & Calanthe angustifolia (Blume) Lindl. \\
\hline & General Distribution: Southeast China to West Malesia \\
\hline & Distribution in Peninsular Malaysia: North of the Peninsula; montane forest \\
\hline & Lifeform: Terrestrial \\
\hline \multirow[t]{4}{*}{32} & Campanulorchis pellipes (Rchb.f. ex Hook.f.) Y. P. Ng \& P. J. Cribb \\
\hline & General Distribution: Thailand to West Malesia \\
\hline & Distribution in Peninsular Malaysia: Widespread; montane forest \\
\hline & Lifeform: Epiphytic \\
\hline \multirow[t]{4}{*}{33} & Ceratostylis ampullacea Kraenzl. \\
\hline & General Distribution: Peninsula Thailand to West Malesia \\
\hline & Distribution in Peninsular Malaysia: Widespread; montane forest \\
\hline & Lifeform: Epiphytic \\
\hline \multirow[t]{4}{*}{34} & Ceratostylis gracilis Blume \\
\hline & General Distribution: West Malesia \\
\hline & Distribution in Peninsular Malaysia: Pahang, Selangor; montane forest \\
\hline & Lifeform: Epiphytic \\
\hline \multirow[t]{4}{*}{35} & Ceratostylis subulata Blume \\
\hline & General Distribution: Tropical Asia to Vanuatu \\
\hline & Distribution in Peninsular Malaysia: Widespread; montane forest \\
\hline & Lifeform: Epiphytic \\
\hline \multirow[t]{4}{*}{36} & Claderia viridiflora Hook.f. \\
\hline & General Distribution: Peninsula Thailand to West \& Central Malesia \\
\hline & Distribution in Peninsular Malaysia: Widespread; montane forest \\
\hline & Lifeform: Epiphytic \\
\hline \multirow[t]{4}{*}{37} & Cleisostoma suffusum (Ridl.) Garay \\
\hline & General Distribution: Peninsular Malaysia to Sumatera, North Borneo \\
\hline & Distribution in Peninsular Malaysia: Perak, Pahang and Malacca; hill forest \\
\hline & Lifeform: Epiphytic \\
\hline \multirow[t]{4}{*}{38} & Coelogyne anceps Hook.f. \\
\hline & General Distribution: Endemic in Peninsular Malaysia \\
\hline & Distribution in Peninsular Malaysia: Gunung Tahan (Perak, Pahang); montane forest \\
\hline & Lifeform: Epiphytic \\
\hline
\end{tabular}


Table 2 (continue)

\begin{tabular}{|c|c|}
\hline No. & Species \\
\hline \multirow[t]{4}{*}{39} & Coelogyne cumingii Lindl. \\
\hline & General Distribution: Indo-China to West Malesia \\
\hline & Distribution in Peninsular Malaysia: Widespread; lowland and hill forest \\
\hline & Lifeform: Epiphytic \\
\hline \multirow[t]{4}{*}{40} & Coelogyne kaliana P.J.Cribb \\
\hline & General Distribution: Endemic in Peninsular Malaysia \\
\hline & Distribution in Peninsular Malaysia: Perak, Pahang and Selangor; montane forest \\
\hline & Lifeform: Epiphytic \\
\hline \multirow[t]{4}{*}{41} & Coelogyne tomentosa Lindl. \\
\hline & General Distribution: Peninsula Thailand to West Malesia \\
\hline & Distribution in Peninsular Malaysia: Perak; montane forest \\
\hline & Lifeform: Epiphytic \\
\hline \multirow[t]{4}{*}{42} & Corybas carinatus (J.J.Sm.) Schltr. \\
\hline & General Distribution: West Malesia \\
\hline & Distribution in Peninsular Malaysia: Perak, Pahang, Johor; montane forest \\
\hline & Lifeform: Terrestrial \\
\hline \multirow[t]{4}{*}{43} & Corymborkis veratrifolia (Reinw.) Blume \\
\hline & General Distribution: Tropical and Subtropical Asia to Pacific \\
\hline & Distribution in Peninsular Malaysia: Widespread; lowland and montane forest \\
\hline & Lifeform: Terrestrial \\
\hline \multirow[t]{4}{*}{44} & Crepidium calophyllum (Rchb.f.) Szlach. \\
\hline & General Distribution: East Nepal to Hainan and Borneo \\
\hline & Distribution in Peninsular Malaysia: Kedah, Kelantan, Pulau Pinang, Johor; hill forest \\
\hline & Lifeform: Terrestrial \\
\hline \multirow[t]{4}{*}{45} & Cryptostylis arachnites (Blume) Hassk. in C.L.Blume \\
\hline & General Distribution: Tropical \& Subtropical Asia to South West Pacific \\
\hline & Distribution in Peninsular Malaysia: Widespread; montane forest \\
\hline & Lifeform: Terrestrial \\
\hline \multirow[t]{4}{*}{46} & Cylindrolobus nutans (Lindl.) J.J.Wood \\
\hline & General Distribution: Thailand to West Malesia \\
\hline & Distribution in Peninsular Malaysia: Widespread; montane forest \\
\hline & Lifeform: Epiphytic \\
\hline \multirow[t]{4}{*}{47} & Cymbidium finlaysonianum Lindl. \\
\hline & General Distribution: Indo-China to Malesia \\
\hline & Distribution in Peninsular Malaysia: Most abundant in the north; lowland forest \\
\hline & Lifeform: Epiphytic \\
\hline \multirow[t]{4}{*}{48} & Dendrobium angustifolium (Blume) Lindl. \\
\hline & General Distribution: Arunachal Pradesh to China and West Malesia \\
\hline & Distribution in Peninsular Malaysia: Widespread; montane forest \\
\hline & Lifeform: Epiphytic \\
\hline \multirow[t]{4}{*}{49} & Dendrobium convexum (Blume) Lindl. \\
\hline & General Distribution: Indo-China to North Queensland \\
\hline & Distribution in Peninsular Malaysia: Gunung Ulu Kali, Selangor; montane forest \\
\hline & Lifeform: Epiphytic \\
\hline \multirow[t]{4}{*}{50} & Dendrobium crumenatum $\mathrm{Sw}$. \\
\hline & General Distribution: Taiwan to Tropical Asia \\
\hline & Distribution in Peninsular Malaysia: Widespread and common; lowland forest \\
\hline & Lifeform: Epiphytic \\
\hline \multirow[t]{4}{*}{51} & Dendrobium derryi Ridl. \\
\hline & General Distribution: West Malesia \\
\hline & Distribution in Peninsular Malaysia: Perak; montane forest \\
\hline & Lifeform: Epiphytic \\
\hline \multirow[t]{4}{*}{52} & Dendrobium geminatum (Blume) Lindl. \\
\hline & General Distribution: West Malesia \\
\hline & Distribution in Peninsular Malaysia: Many localities on high exposed mountain ridges \\
\hline & Lifeform: Epiphytic \\
\hline
\end{tabular}


Farah Alia Nordin, Ahmad Sofiman Othman, Nur Asyikin Zainudin, Nur 'Atiqah Khalil, Najidah Asi, Afifah Azmi, Khairul Nasirudin Abu Mangsor, Mohd Sukor Harun and Khairul Faizee Mohd Zin

Table 2 (continue)

\begin{tabular}{|c|c|}
\hline No. & Species \\
\hline \multirow[t]{4}{*}{53} & Dendrobium indivisum (Blume) Miq. \\
\hline & General Distribution: Bangladesh to Malesia \\
\hline & Distribution in Peninsular Malaysia: Widespread; lowland forest \\
\hline & Lifeform: Epiphytic \\
\hline \multirow[t]{4}{*}{54} & Dendrobium kelsallii Ridl. \\
\hline & General Distribution: Endemic in Peninsular Malaysia \\
\hline & Distribution in Peninsular Malaysia: Johor; lowland forest \\
\hline & Lifeform: Epiphytic \\
\hline \multirow[t]{4}{*}{55} & Dendrobium lamellatum (Blume) Lindl. \\
\hline & General Distribution: Java and Peninsular Malaysia \\
\hline & Distribution in Peninsular Malaysia: Widespread but uncommon; lowland forest \\
\hline & Lifeform: Epiphytic \\
\hline \multirow[t]{4}{*}{56} & Dendrobium leonis (Lindl.) Rchb.f. in W.G.Walpers \\
\hline & General Distribution: Indo-China to West Malesia \\
\hline & Distribution in Peninsular Malaysia: Widespread and common; lowland forest \\
\hline & Lifeform: Epiphytic and lithophytic \\
\hline \multirow[t]{4}{*}{57} & Dendrobium longipes Hook.f. \\
\hline & General Distribution: Peninsular Malaysia to West Sumatra \\
\hline & Distribution in Peninsular Malaysia: Many localities on high exposed mountain ridges \\
\hline & Lifeform: Epiphytic \\
\hline \multirow[t]{4}{*}{58} & Dendrobium macropodum Hook.f. \\
\hline & General Distribution: West Malesia \\
\hline & Distribution in Peninsular Malaysia: Many localities on high exposed mountain ridges \\
\hline & Lifeform: Epiphytic \\
\hline \multirow[t]{4}{*}{59} & Dendrobium mannii Ridl. \\
\hline & General Distribution: Arunachal Pradesh to Peninsular Malaysia \\
\hline & Distribution in Peninsular Malaysia: Malacca and Johor; lowland and hill forest \\
\hline & Lifeform: Epiphytic \\
\hline \multirow[t]{4}{*}{60} & Dendrobium metachilinum Rchb.f. \\
\hline & General Distribution: Peninsula Thailand to West Malesia, Maluku (Ambon) \\
\hline & Distribution in Peninsular Malaysia: Common in south of Peninsular Malaysia; lowland forest \\
\hline & Lifeform: Epiphytic \\
\hline \multirow[t]{4}{*}{61} & Dendrobium pachyglossum E.C.Parish \& Rchb.f. \\
\hline & General Distribution: Indo-China to Peninsular Malaysia, Borneo (Sarawak) \\
\hline & Distribution in Peninsular Malaysia: Several localities; montane forest \\
\hline & Lifeform: Epiphytic \\
\hline \multirow[t]{4}{*}{62} & Dendrobium uniflorum Griff. \\
\hline & General Distribution: Indo-China to West and Central Malesia \\
\hline & Distribution in Peninsular Malaysia: Widespread; lowland forest \\
\hline & Lifeform: Epiphytic \\
\hline \multirow[t]{4}{*}{63} & Dendrobium villosulum Wall. ex Lindl. \\
\hline & General Distribution: Thailand, Peninsular Malaysia (P. Pinang), Borneo \\
\hline & Distribution in Peninsular Malaysia: Widespread; lowland and montane forest \\
\hline & Lifeform: Epiphytic \\
\hline \multirow[t]{4}{*}{64} & Dendrochilun linearifolium Hook.f. \\
\hline & General Distribution: Peninsular Malaysia to Sumatra \\
\hline & Distribution in Peninsular Malaysia: Quite widespread; montane forest \\
\hline & Lifeform: Epiphytic \\
\hline \multirow[t]{4}{*}{65} & Dendrochilum longifolium Rchb.f. \\
\hline & General Distribution: Indo-China to Papuasia \\
\hline & Distribution in Peninsular Malaysia: Pahang southward; lowland forest \\
\hline & Lifeform: Epiphytic \\
\hline \multirow[t]{4}{*}{66} & Dipodium conduplicatum J.J.Sm. \\
\hline & General Distribution: Peninsular Malaysia to North and West Sumatra \\
\hline & Distribution in Peninsular Malaysia: Pahang, Johor; montane forest \\
\hline & Lifeform: Terrestrial, sometimes climbing \\
\hline
\end{tabular}


Table 2 (continue)

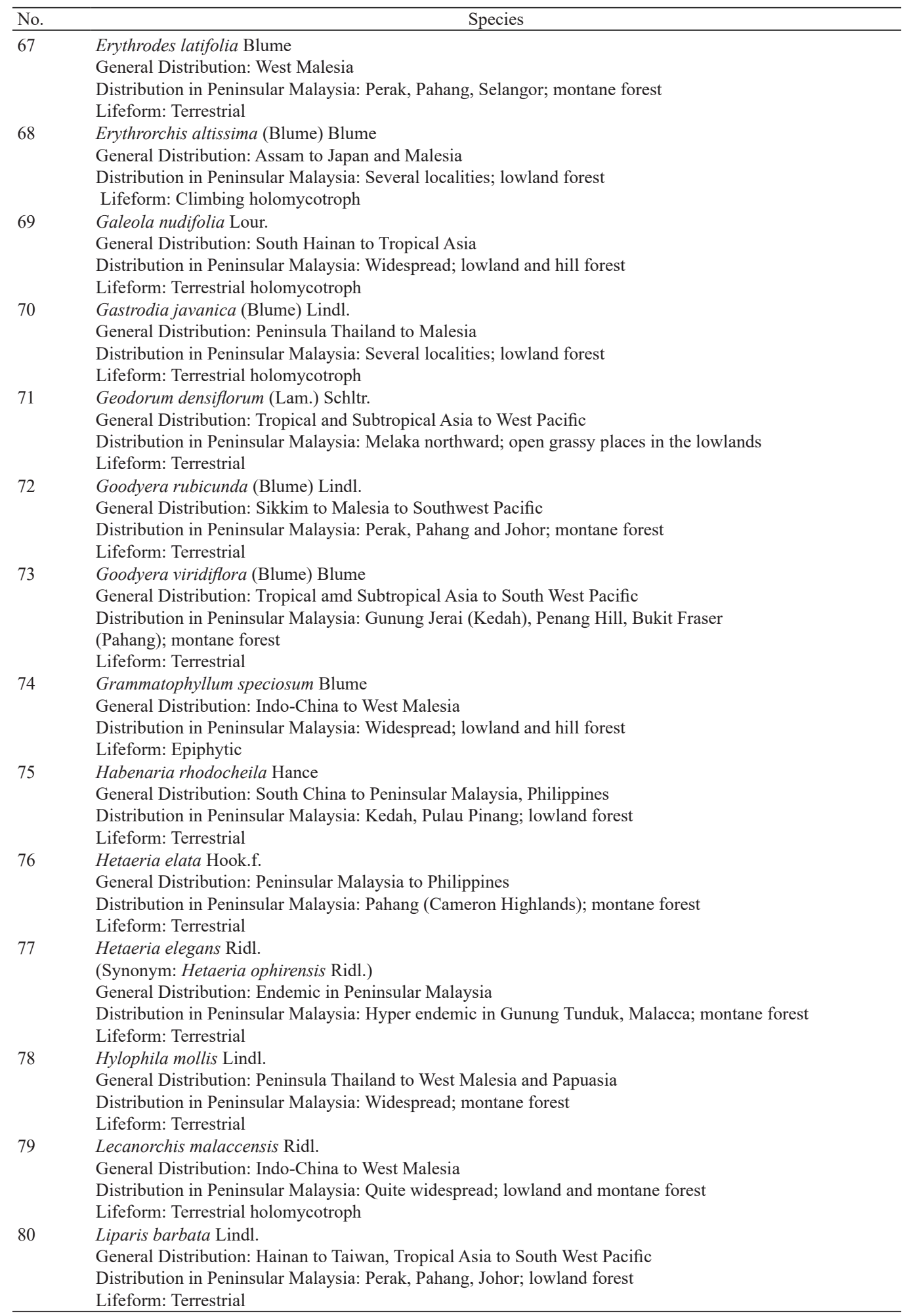


Farah Alia Nordin, Ahmad Sofiman Othman, Nur Asyikin Zainudin, Nur 'Atiqah Khalil, Najidah Asi, Afifah Azmi, Khairul Nasirudin Abu Mangsor, Mohd Sukor Harun and Khairul Faizee Mohd Zin

Table 2 (continue)

\begin{tabular}{|c|c|}
\hline No. & Species \\
\hline \multirow[t]{5}{*}{81} & Liparis elegans Lindl. \\
\hline & (Synonym: Stichorkis elegans (Lindl.) Marg., Szlach. \& Kulak) \\
\hline & General Distribution: Nicobar Island to Hainan and South West Pacific \\
\hline & Distribution in Peninsular Malaysia: Widespread; lowland and hills to $1000 \mathrm{~m}$ \\
\hline & Lifeform: Epiphytic or lithophytic \\
\hline \multirow[t]{4}{*}{82} & Liparis maingayi (Hook.f.) Ridl. \\
\hline & General Distribution: Peninsular Malaysia to West Sumatera \\
\hline & Distribution in Peninsular Malaysia: Kedah, Pulau Pinang, Perak, Johor; hill forest \\
\hline & Lifeform: Epiphytic \\
\hline \multirow[t]{4}{*}{83} & Liparis viridicallus Holttum \\
\hline & General Distribution: West Malesia to Philippines \\
\hline & Distribution in Peninsular Malaysia: Pahang (Fraser's Hill and Gunung Ulu Kali); montane forest \\
\hline & Lifeform: Terrestrial or lithophytic \\
\hline \multirow[t]{3}{*}{84} & Luisia sp. \\
\hline & Distribution in Peninsular Malaysia: Gunung Ledang; hill forest \\
\hline & Lifeform: Epiphytic \\
\hline \multirow[t]{4}{*}{85} & Macodes petola (Blume) Lindl. \\
\hline & General Distribution: South Japan, Peninsula Thailand to West and Central Malesia \\
\hline & Distribution in Peninsular Malaysia: Pulau Pinang southward; damp lowland and hill forest \\
\hline & Lifeform: Terrestrial \\
\hline \multirow[t]{4}{*}{86} & Neuwiedia griffithii Rchb.f. \\
\hline & General Distribution: Vietnam, Malaya to North Sumatra \\
\hline & $\begin{array}{l}\text { Distribution in Peninsular Malaysia: Pahang, Selangor, Negeri Sembilan, Malacca, and Johor; damp lowland } \\
\text { forest }\end{array}$ \\
\hline & Lifeform: Terrestrial \\
\hline \multirow[t]{4}{*}{87} & Neuwiedia veratrifolia Blume \\
\hline & General Distribution: Malesia to Vanuatu \\
\hline & Distribution in Peninsular Malaysia: Pulau Pinang southward; hill and montane forest \\
\hline & Lifeform: Terrestrial \\
\hline \multirow[t]{4}{*}{88} & Oberonia bertoldii King \& Pantl. \\
\hline & General Distribution: Endemic in Peninsular Malaysia \\
\hline & Distribution in Peninsular Malaysia: Perak, Pahang, Selangor and Johor; lowland forest \\
\hline & Lifeform: Epiphytic \\
\hline \multirow[t]{4}{*}{89} & Paphiopedilum barbatum (Lindl.) Pfitzer \\
\hline & General Distribution: Peninsula Thailand to North Sumatra \\
\hline & Distribution in Peninsular Malaysia: Widespread; open grassy or rocky places in the mountains \\
\hline & Lifeform: Terrestrial \\
\hline \multirow[t]{5}{*}{90} & Peristylus maingayi (King \& Pantl.) J.J.Wood \& Ormerod \\
\hline & (Synonym: Peristylus candidus J.J.Sm.) \\
\hline & General Distribution: South Indo-China to North Queensland \\
\hline & Distribution in Peninsular Malaysia: Commoner in the south of Peninsular Malaysia; montane forest \\
\hline & Lifeform: Terrestrial \\
\hline \multirow[t]{4}{*}{91} & Peristylus monticola (Ridl.) Seidenf. \\
\hline & General Distribution: Andaman Island, Malesia to New Guinea \\
\hline & Distribution in Peninsular Malaysia: Gunung Jerai (Kedah), Gunung Ledang (Johor); montane forest \\
\hline & Lifeform: Terrestrial \\
\hline \multirow[t]{5}{*}{92} & Phalaenopsis deliciosa Rchb.f. \\
\hline & (Synonym: Kingidium deliciosum (Rchb.f.) H.R.Sweet) \\
\hline & General Distribution: India to China to Malesia \\
\hline & Distribution in Peninsular Malaysia: Widespread but not common; lowland and hill forest \\
\hline & Lifeform: Epiphytic \\
\hline \multirow[t]{4}{*}{93} & Phalaenopsis fuscata Rchb.f. \\
\hline & General Distribution: Peninsular Malaysia to Philippines \\
\hline & Distribution in Peninsular Malaysia: Pahang and Johor; lowland forest \\
\hline & Lifeform: Epiphytic \\
\hline
\end{tabular}


Table 2 (continue)

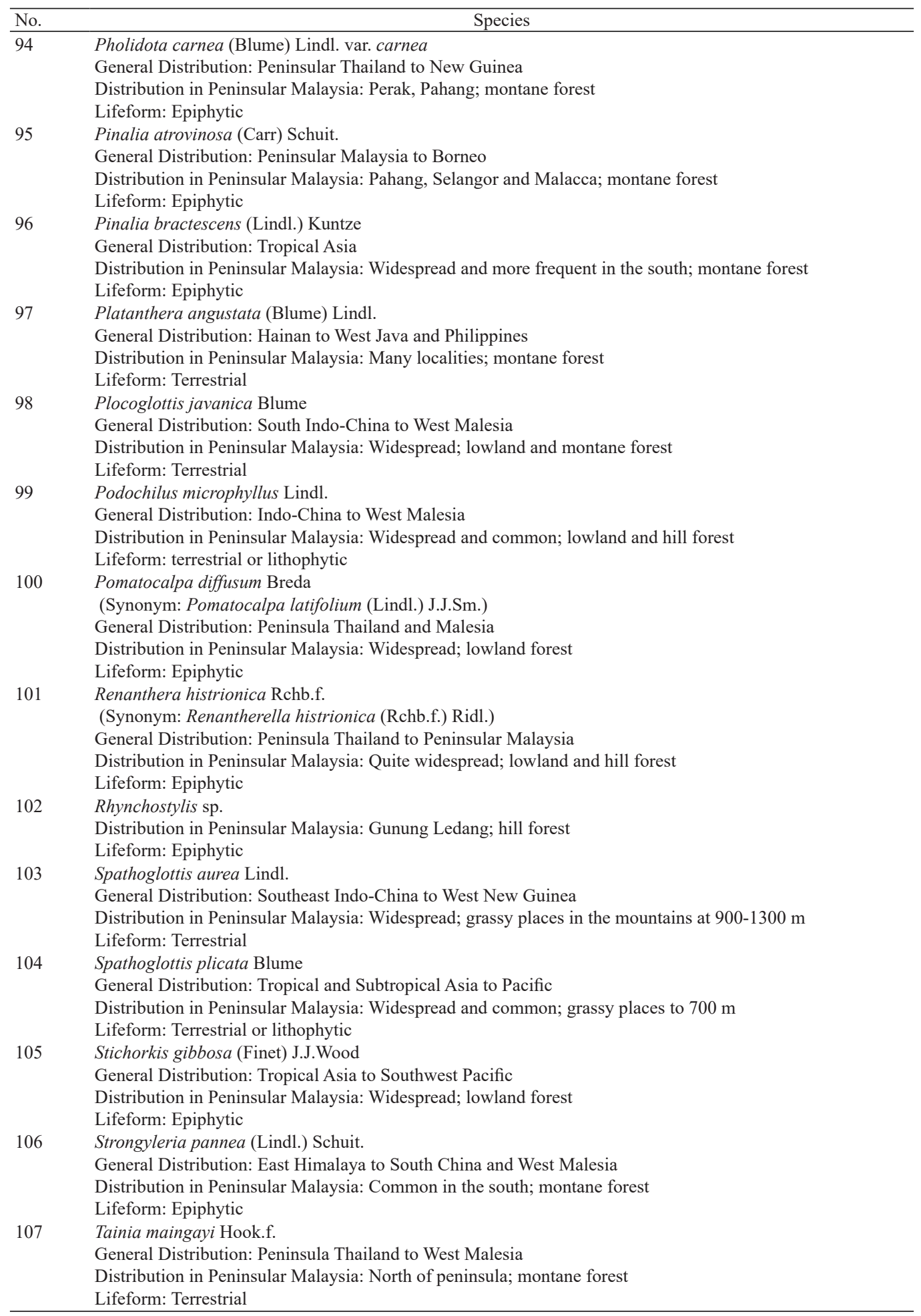


Farah Alia Nordin, Ahmad Sofiman Othman, Nur Asyikin Zainudin, Nur 'Atiqah Khalil, Najidah Asi, Afifah Azmi, Khairul Nasirudin Abu Mangsor, Mohd Sukor Harun and Khairul Faizee Mohd Zin

Table 2 (continue)

\begin{tabular}{|c|c|}
\hline No. & Species \\
\hline \multirow[t]{4}{*}{108} & Thecopus maingayi (Hook.f.) Seidenf. \\
\hline & General Distribution: South Indo-China to West Malesia \\
\hline & Distribution in Peninsular Malaysia: Malacca; lowland forest \\
\hline & Lifeform: Epiphytic \\
\hline \multirow[t]{4}{*}{109} & Tainia speciosa Blume \\
\hline & General Distribution: Thailand to West Malesia \\
\hline & Distribution in Peninsular Malaysia: Widespread; montane forest \\
\hline & Lifeform: Terrestrial \\
\hline \multirow[t]{3}{*}{110} & Thrixspermum sp. \\
\hline & Distribution in Peninsular Malaysia: Gunung Ledang; montane forest \\
\hline & Lifeform: Epiphytic \\
\hline \multirow[t]{4}{*}{111} & Trichotosia ferox Blume \\
\hline & General Distribution: Thailand to West and South Malesia \\
\hline & Distribution in Peninsular Malaysia: Widespread; montane forest \\
\hline & Lifeform: Epiphytic \\
\hline \multirow[t]{4}{*}{112} & Trichotosia gracilis (Hook.f.) Kraenzl. \\
\hline & General Distribution: Indo-China to West Malesia \\
\hline & Distribution in Peninsular Malaysia: Widespread; montane forest \\
\hline & Lifeform: Epiphytic \\
\hline \multirow[t]{4}{*}{113} & Trichotosia pauciflora Blume \\
\hline & General Distribution: Thailand to West Malesia and Lesser Sunda Island (Bali) \\
\hline & Distribution in Peninsular Malaysia: Widespread; montane forest \\
\hline & Lifeform: Epiphytic \\
\hline \multirow[t]{4}{*}{114} & Trichotosia poculata (Ridl.) Kraenzl. \\
\hline & General Distribution: West Malesia \\
\hline & Distribution in Peninsular Malaysia: Widespread; montane forest \\
\hline & Lifeform: Epiphytic \\
\hline \multirow[t]{4}{*}{115} & Trichotosia velutina (Lodd. ex Lindl.) Kraenzl. \\
\hline & General Distribution: Arunachal Pradesh to West Malesia \\
\hline & Distribution in Peninsular Malaysia: Widespread and common; montane forest \\
\hline & Lifeform: Epiphytic \\
\hline \multirow[t]{4}{*}{116} & Trichotosia vestita (Wall. ex Lindl.) Kraenzl. \\
\hline & General Distribution: West Malesia \\
\hline & Distribution in Peninsular Malaysia: Widespread; montane forest \\
\hline & Lifeform: Epiphytic \\
\hline \multirow[t]{4}{*}{117} & Tropidia angulosa (Lindl.) Blume \\
\hline & General Distribution: Bhutan to South China and Lesser Sunda Island (Bali) \\
\hline & Distribution in Peninsular Malaysia: Perak; lowland and montane forest \\
\hline & Lifeform: Terrestrial \\
\hline \multirow[t]{4}{*}{118} & Tropidia curculigoides Lindl. \\
\hline & General Distribution: East Himalaya to South China and West and Central Malesia \\
\hline & Distribution in Peninsular Malaysia: Widespread; lowland and montane forest \\
\hline & Lifeform: Terrestrial \\
\hline \multirow[t]{4}{*}{119} & Vanilla griffithii Rchb.f. \\
\hline & General Distribution: Peninsula Thailand to West Malesia \\
\hline & Distribution in Peninsular Malaysia: Widespread and common; lowland and montane forest \\
\hline & Lifeform: Climber \\
\hline \multirow[t]{4}{*}{120} & Zeuxine affinis (Lindl.) Benth. ex Hook.f. \\
\hline & General Distribution: Indian Subcontinent to Nansei-shoto and Peninsular Malaysia \\
\hline & Distribution in Peninsular Malaysia: Gunung Jerai (Kedah), Penang Hill; montane forest \\
\hline & Lifeform: Terrestrial \\
\hline \multirow[t]{4}{*}{121} & Zeuxine gracilis (Breda) Blume \\
\hline & General Distribution: India to West Malesia \\
\hline & Distribution in Peninsular Malaysia: Kedah, Penang, Pahang; montane forest \\
\hline & Lifeform: Terrestrial \\
\hline
\end{tabular}




\section{DISCUSSION}

One of the interesting findings from this study is the discovery of a peculiar jewel orchid species from the genus Anoectochilus (Anoectochilus sp., NAJ17). Few individuals were observed to dwell dispersedly on the dampened ground rich in humus, in between the steep and narrow route from Gunung Tunduk towards the misty valley commencing the peak of Gunung Ledang (Figure 2). The population is very small and rare, growing among the more common dark-burgundy Anoectochilus albolineatus. The leaf is lime-green with interconnecting golden veins, which immediately can be easily mistaken with the velvety Macodes petola. However, during the visit, this unknown species of Anoectochilus is not in its flowering state that to proceed with taxonomic determination is a challenge. Vegetatively, the species closely resembles Anoectochilus roxburghii which is native to Indo-China. However, any detail on this discovery is put on hold until new information comes into light.

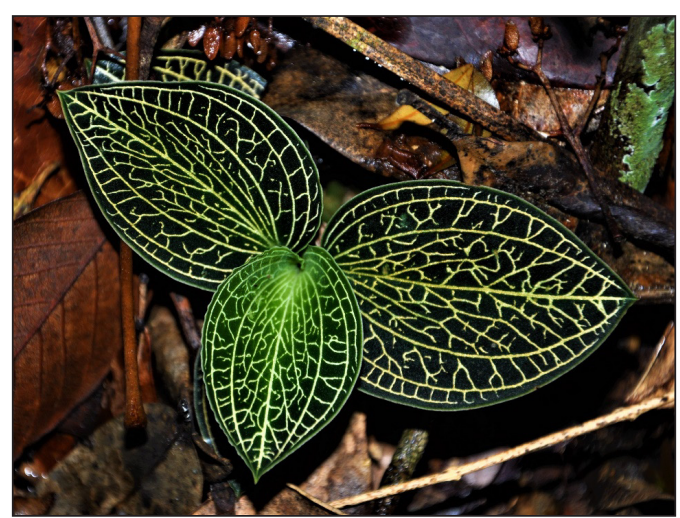

Figure 2. The undetermined orchid species from Gunung Ledang (Anoectochilus sp., NJ17). Photo by Nordin, F. A.
None of the two hyper-endemic species which have been listed previously by Turner (1995) were encountered in this study, proven by their narrow distribution and rarity. Hetaeria elegans, previously known as $H$. ophirensis, was discovered, and described by Ridley from Gunung Tunduk in 1908. Seidenfaden and Wood (1992) stated that $H$. elegans may be conspecific with $H$. elata, the sister species that was found to grow quite abundantly on the rich humus along the route to the bare rocky area in Gunung Tunduk at about $1200 \mathrm{~m}$ above sea level. Thus, more research needs to be done to resolve the taxonomic questions between the two species.

In a nutshell, Gunung Ledang was proven to be rich and diverse with its orchid flora, with Ridley's historical routes via Gunung Tunduk, the Lagenda Trail via Batu Orkid, and the peak of Gunung Ledang offer myriads of interesting discoveries. Some of the enchanting beauties are shown in Figure 3A-L. The higher peaks of Gunung Ledang were occasionally clouded in mist during the day, making them as desirable habitats for the montane orchid species. Meanwhile, the Ulu Jementah Trail via Jeram Tinggi worth the exploration, however fewer orchid species were counted at the lower levels of the forest. The route begins to be consistently rich with orchid species as the ascent commences Gunung Mahligai towards the peak of Gunung Ledang.

By contribution, this paper provides an updated account on the diversity of orchids in Gunung Ledang, listing 122 species of orchids, of which eight are endemic to 
Farah Alia Nordin, Ahmad Sofiman Othman, Nur Asyikin Zainudin, Nur 'Atiqah Khalil, Najidah Asi, Afifah Azmi, Khairul Nasirudin Abu Mangsor, Mohd Sukor Harun and Khairul Faizee Mohd Zin

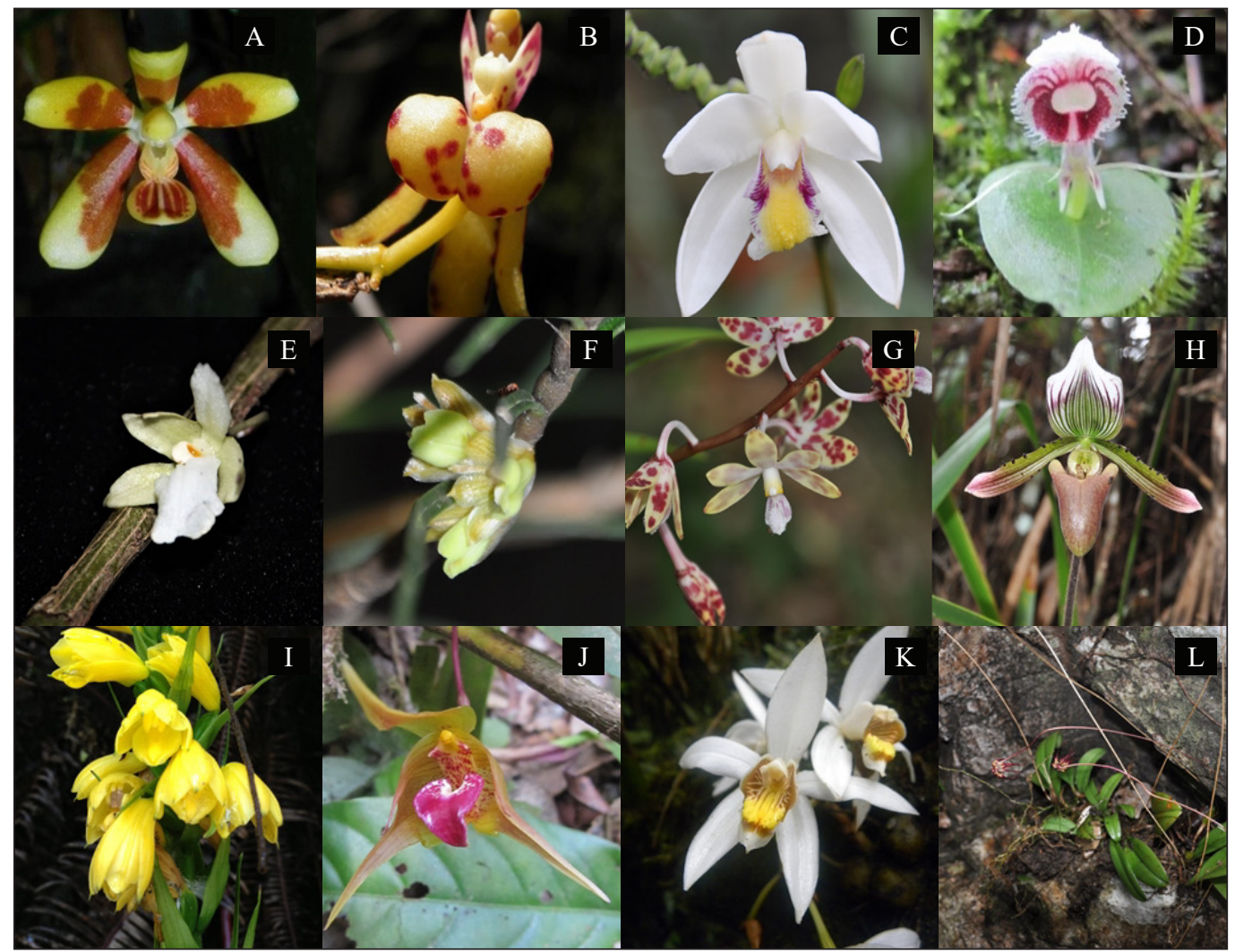

Figure 3. Myriads of orchid species from Gunung Ledang, (A) Phalaenopsis fuscata, (B) Renanthera histrionica, (C) Bromheadia finlaysoniana, (D) Corybas carinatus, (E) Dendrobium derryi, (F) Dendrobium villosulum, (G) Dipodium conduplicatum, (H) Paphiopedilum barbatum, (I) Neuwiedia veratrifolia, (J) Bulbophyllum uniflorum, (K) Coelogyne kaliana, and (L) Bulbophyllum gracillimum. Photos by Nordin, F. A.

Peninsular Malaysia, two are hyper-endemic known only from Gunung Ledang, 30 were recognised as new records, and one species needs further taxonomic clarification.

\section{CONCLUSION}

Gunung Ledang exhibits a great diversity of orchids with Ridley's historical routes were revisited and new captivating routes were explored. The 122 species in 62 genera portrayed the exceptionally rich orchid flora found on the mountain region. The decision to gazette the forests of Gunung Ledang as a national park has ensured the conservation of the rich and unique biodiversity represented in these still pristine forest areas, and especially the survival of the notable orchids as floristic heritage.

\section{ACKNOWLEDGEMENTS}

The authors would like to express our deepest gratitude to Johor National Park Corporation for the facilities and collaborative assistance provided, to School of Biological Sciences, Universiti Sains Malaysia, to the administrative and staff of Gunung Ledang National Park and Jabatan Pertahanan Awam Malaysia for 
the logistics and field assistance provided during the study conducted. This project is funded by the USM Research University Grant (PBIOLOGY 843082) awarded to the second author.

\section{REFERENCES}

Comber, J. B. (2001). Orchids of Sumatra. Natural History Publications.

Go, R., Abdullah, J. O., Nordin, F. A., \& Mat Isa, S. F. (2015). Orchids in the montane forests of Peninsular Malaysia. Penerbit Universiti Putra Malaysia.

Holttum, R. E. (1964). A revised flora of Malaya: Orchids of Malaya (Vol. 1, $3^{\text {rd }}$ ed.). Government Printing Office.

Ong, P. T., O’Byrne, P., Saw, L. G., \& Chung, R. C. K. (2017). Checklist of orchids of Peninsular Malaysia. Forest Research Institute Malaysia.
Ridley, H. N. (1901). The flora of Mount Ophir. Journal of the Straits Branch of the Royal Asiatic Society, 35, 1-28.

Ridley, H. N. (1907). Materials for a flora of the Malayan Peninsula (Vol. 2). Methodist Publishing House.

Ridley, H. N. (1924). The flora of the Malay Peninsula: Monocotyledones (Vol. 4). L. Reeve \& Co. Ltd.

Seidenfaden, G., \& Smitinand, T. (1959). The orchids of Thailand: A preliminary list. The Siam Society.

Seidenfaden, G., \& Wood, J. J. (1992). The orchids of Peninsular Malaysia and Singapore. Olsen $\&$ Olsen in association with The Royal Botanic Gardens, Kew \& Singapore Botanic Gardens.

Turner, I. M. (1995). A catalogue of the vascular plants of Malaya: Orchidaceae. The Gardens' Bulletin Singapore, 47(2), 559-620.

World Checklist of Selected Plant Families. (2020). World checklist of selected plant families. WCSP. http://wcsp.science.kew.org/ 
